\title{
A Fluent Calculus Semantics for ADL with Plan Constraints
}

\author{
Conrad Drescher and Michael Thielscher \\ Department of Computer Science, \\ Dresden University of Technology \\ Nöthnitzer Str. 46, 01187 Dresden, Germany
}

\begin{abstract}
Plan constraints are the most recent addition to the ever growing Planning Domain Definition Language (PDDL). In this work we consider the PDDL fragment consisting of basic ADL extended by plan constraints. We provide a purely declarative semantics for this fragment by interpreting it in the basic Fluent Calculus. We thus obtain a logical semantics for this fragment of PDDL instead of the usual meta-theoretical state transition semantics.
\end{abstract}

\section{Introduction}

Research in specialized planning languages originates with STRIPS [1]. Over the years this basic language has seen numerous extensions: first to the language ADL [2] and then to PDDL [3], the ever growing language that underlies the annual planning competitions. The new feature in the most recent version PDDL 3.0 [4] are plan constraints that allow to express both requirements and preferences with regard to plan quality.

Traditionally the semantics of planning languages is given in terms of state transitions. There also is a parallel line of research that seeks to provide a logical semantics for planning languages. Such complementary semantics exist for STRIPS [1,5] and ADL $[6,7]$. The recent works $[8,9]$ aim at successively covering all of the semantics of PDDL 2.1 [10].

In this work we provide a purely declarative semantics for the fragment of PDDL 3.0 consisting of basic ADL and plan constraints. We do so by interpreting this fragment in the basic Fluent Calculus. The resulting system is both natural and expressive.

\section{Preliminaries}

In this section we recall the theoretical basis upon which our work rests. We start by recalling the basics of Fluent Calculus. Then we identify the fragment of PDDL under consideration - ADL with plan constraints.

\subsection{Fluent Calculus}

The Fluent Calculus [11] can be seen as a modern extension of the classical Situation Calculus [12]. One of the major differences between Fluent and Situation Calculus is 
that the former is action-centered while the latter is fluent-centered - at least in the popular version based on Reiter's successor state axioms [12]. That is to say, in Fluent Calculus we specify for each action the effects it has while a successor state axiom specifies for a fluent by which actions it is affected. Thus Fluent Calculus arguably is closer to current planning languages, which are also action-centered.

Our work uses a reformulation of the basic Fluent Calculus in a recently proposed unifying action calculus (UAC) [13] that allows us to keep the technical overhead to a minimum. A comprehensive treatment of the classical Fluent Calculus - which also captures the notion of state, i.e. of collections of fluents — can be found in [11].

Unifying Action Calculus The UAC has been introduced with the stated goal of bundling research efforts in the reasoning about action community; it has been shown to encompass the Event, Fluent, and Situation Calculus, as well as planning languages such as ADL.

Formally, the UAC is based on many-sorted first order logic with equality and the four sorts TIME, FLUENT, OBJECT, and ACTION. ${ }^{1}$ Fluents are reified, i.e. modeled as terms, and the predicate Holds : FLUENT $\times$ TIME is used to indicate whether a particular fluent evaluates to true at a particular time. For axiomatizing action preconditions the predicate Poss : ACTION $\times$ TIME $\times$ TIME is used. ${ }^{2}$ There are only finitely many function symbols into sorts FLUENT and ACTION, respectively.

The UAC abstracts from a particular time structure. It can be instantiated, e.g., by the natural numbers that serve as the linear time structure of the Event Calculus, or by situations that provide the branching time structure of the Fluent and Situation Calculus.

Fluent Calculus Domains Fluent Calculus domains are axiomatized in the UAC with the help of the following formula types:

\section{Definition 1 (Basic Formulas).}

For $\overline{\mathbf{s}}$, a sequence of variables of sort TIME, a state formula $\Phi[\overline{\mathbf{s}}]$ in $\overline{\mathbf{s}}$ is a first-order formula with free variables $\overline{\mathbf{s}}$ and where

- for each occurrence of Holds $(f, s)$ we have $s \in \overline{\mathbf{s}}$;

- predicate Poss does not occur.

Let $A$ be a function into sort ACTION.

- A domain constraint is a state formula in $s$ :

$$
(\forall s) \delta[s] .
$$

- A precondition axiom is of the form

$$
(\forall) \operatorname{Poss}\left(A(\overline{\mathbf{x}}), s_{1}, s_{2}\right) \equiv \pi_{A}\left[s_{1}\right] \wedge s_{2}=\operatorname{Do}\left(A(\overline{\mathbf{x}}), s_{1}\right),
$$

where $\pi_{A}\left[s_{1}\right]$ is a state formula in $s_{1}$ with free variables among $s_{1}, \overline{\mathbf{x}}^{3}$

\footnotetext{
${ }^{1}$ By convention variable symbols $s, f, x$, and $a$ are used for terms of sort TIME, FLUENT, OBJECT, and ACTION, respectively.

${ }^{2}$ Having two arguments of sort TIME allows to model actions with duration or indirect effects.

${ }^{3}$ By $(\forall) \varphi$ we denote the universal closure of $\varphi$.
} 
- An effect axiom is of the form

$(\forall) \operatorname{Poss}(A(\overline{\mathbf{x}}), s, t) \supset$
$\bigvee_{k}\left(\exists \overline{\mathbf{y}}_{k}\right)\left(\Phi_{k}[s] \wedge(\forall f)\left[\bigvee_{i} f=f_{k i} \vee \operatorname{Holds}(f, s) \wedge \bigwedge_{j} f \neq g_{k j} \equiv \operatorname{Holds}(f, t)\right]\right)$

The $f_{k i}$ and $g_{k j}$ are fluent terms with variables among $\overline{\mathbf{x}}, \overline{\mathbf{y}}_{k}$ and denote the positive and negative effects, respectively. The $\Phi_{k}$ are state formulas in $s$ with free variables among $s, \overline{\mathbf{x}}, \overline{\mathbf{y}}$ and represent conditions under which the effects materialize. Positive and negative action effects are subject to a natural consistency assumption, namely, we require that

$$
\bigwedge_{i} \bigwedge_{j} f_{k i} \neq g_{k j}
$$

holds for all $k=1, \ldots, n$.

- An initial state axiom is a state formula in the least element $S_{0}$ of sort TIME.

- Foundational axioms $\Sigma_{\text {aux }}$ contain a first order axiomatization of situations (the underlying time structure). It is based on two functions into sort TIME; the constant $S_{0}$ denotes the initial situation and the function Do of sort ACTION $\times$ TIME is used to construct successor situations:

$$
\begin{aligned}
& (\forall) \operatorname{Do}\left(a_{1}, s_{1}\right)=\operatorname{Do}\left(a_{2}, s_{2}\right) \equiv a_{1}=a_{2} \wedge s_{1}=s_{2} \\
& (\forall) \neg s \sqsubset S_{0} \\
& (\forall) s \sqsubset D o\left(a, s^{\prime}\right) \equiv s \sqsubseteq s^{\prime} \\
& \phi\left[S_{0}\right] \wedge(\forall s, a)(\phi[s] \supset \phi[D o(a, s)]) \supset\left(\forall s^{\prime}\right) \phi\left[s^{\prime}\right]
\end{aligned}
$$

where in the axiom scheme on the last line $\phi$ ranges over all state formulas in $s$, with only s free. Foundational axioms $\Sigma_{\text {aux }}$ also contain unique name axioms for sorts ACTION and FLUENT; that is, an axiom of the form

$$
(\forall \overline{\mathbf{x}} \forall \overline{\mathbf{y}}) \bigwedge_{i=1 . . n-1} \bigwedge_{j=i+1 . . n} T_{i}(\overline{\mathbf{x}}) \neq T_{j}(\overline{\mathbf{y}}) \wedge \bigwedge_{i=1 . . n} T_{i}(\overline{\mathbf{x}})=T_{i}(\overline{\mathbf{y}}) \supset \overline{\mathbf{x}}=\overline{\mathbf{y}}
$$

where the $T_{i}$ range over all function symbols of the respective sorts. For dealing with arithmetic later on we introduce the sort NUMBER and include an axiomatization of Presburger arithmetic.

Definition 2 (Domain Axiomatizations). A domain axiomatization $\Sigma$ consists of a set $\Sigma_{\text {Poss }}$ of precondition-, and a set $\Sigma_{\text {Effects }}$ of effect axioms, each containing one axiom for every function into sort ACTION, along with a finite set of domain constraints $\Sigma_{d c}$, a finite set of initial state axioms $\Sigma_{\text {Init }}$, and foundational axioms $\Sigma_{\text {aux }}$.

Let us illustrate all the introduced notions by an axiomatization of the familiar blocks world domain: 
Example 1 (Blocks World Axiomatization). The precondition of moving a block from some location $x$ to location $y$ is expressed by the following axiom:

$$
\begin{aligned}
& (\forall) \operatorname{Poss}\left(\operatorname{Move}\left(\operatorname{block}_{1}, x, y\right), s_{1}, s_{2}\right) \equiv \\
& \operatorname{Holds}\left(\mathrm{On}\left(\operatorname{block}_{1}, x\right), s_{1}\right) \wedge x \neq y \wedge \\
& \left(\neg \exists \text { block }_{2}\right) \operatorname{Holds}\left(\mathrm{On}\left(\text { block }_{2}, \text { block }_{1}\right), s_{1}\right) \wedge \\
& \left(\neg \exists \text { block }_{3}\right)\left(\operatorname{Holds}\left(\mathrm{On}\left(\mathrm{block}_{3}, y\right), s_{1}\right) \vee y=\text { Table }\right) \wedge \\
& s_{2}=\operatorname{Do}\left(\operatorname{Move}\left(\operatorname{block}_{1}, x, y\right), s_{1}\right) \text {. }
\end{aligned}
$$

The effects of moving a block are axiomatized as follows:

$$
\begin{aligned}
& (\forall) \operatorname{Poss}\left(\operatorname{Move}\left(\operatorname{block}_{1}, x, y\right), s_{1}, s_{2}\right) \supset \\
& \quad\left[(\forall f)\left(f=\operatorname{On}\left(\operatorname{block}_{1}, y\right) \vee\left(\operatorname{Holds}\left(f, s_{1}\right) \wedge f \neq \operatorname{On}\left(\operatorname{block}_{1}, x\right)\right)\right) \equiv \operatorname{Holds}\left(f, s_{2}\right)\right] .
\end{aligned}
$$

The following domain constraint expresses the fact that every block is situated at exactly one location: ${ }^{4}$

$$
(\exists ! y) \operatorname{Holds}(\operatorname{On}(x, y), s) .
$$

Finally, suppose that the following axiom describes what is known about the initial situation:

$$
\left.(\forall f) \operatorname{Holds}\left(f, S_{0}\right) \equiv f=\text { On }\left(\text { Block }_{1} \text {, Table }\right) \vee f=\text { On(Block } 2 \text {, Table }\right) .
$$

It can be easily verified that this axiomatization, together with the unique name axioms for the blocks and the table, entails

$$
\left.\operatorname{Holds}\left(\operatorname{On}\left(\text { Block }_{2}, \text { Block }_{1}\right) \text {, Do(Move }\left(\text { Block }_{2} \text {, Table, Block } 1\right), S_{0}\right)\right) \text {. }
$$

\section{ADL with Plan Constraints}

In this section we introduce the fragment of PDDL 3.0 that we consider in this work - ADL with plan constraints. For a general introduction to action languages based on state transition semantics the reader is referred to [14].

\subsection{ADL}

ADL has originally been introduced to cover the expressive middle-ground between STRIPS and the Situation Calculus. It still plays an important role in the sequential, deterministic part of the international planning competitions.

Definition 3 (ADL Signature). An ADL signature is based on a finite set of types, where types may also be defined as unions of other types. The basic type ОВJЕCT is always included. The signature then contains a finite set of typed constants $\mathcal{C}$ and typed variables $\mathcal{V}$. It also includes a finite set of typed fluents $\mathcal{F}$ of arity $\geq 0$ and likewise a finite set of typed operator names $\mathcal{A}$ with associated arity.

\footnotetext{
${ }^{4} \mathrm{By} \exists ! x \phi[x]$ we abbreviate the first order formula expressing that there is exactly one $x$ such that $\phi[x]$.
} 
Planning problems are expressed in ADL with the help of the following constructs:

\section{Definition 4 (Basic ADL Constructs).}

- A state formula $\phi[\overline{\mathbf{x}}]$ is a first order formula with free variables among $\overline{\mathbf{x}}$ containing as atoms only fluents $F(\overline{\mathbf{t}})$ and equalities $\overline{\mathbf{t}}_{1}=\overline{\mathbf{t}}_{2}$.

- An effect formula is the universal closure of a first order conjunction built from the following inductively defined admissible components:

- fluent literals $F(\overline{\mathbf{t}})$ and $\neg F(\overline{\mathbf{t}})$ are admissible;

- if $\phi$ and $\psi$ are admissible then the conjunction $\phi \wedge \psi$ and the universally quantified $(\forall \overline{\mathbf{x}}) \phi$ are;

- if $\phi$ is a state formula and $\psi$ is admissible with no occurrence of $\Rightarrow$ or $\forall$ then $\phi \Rightarrow \psi$ is.

- For an operator name $A \in \mathcal{A}$ the $\mathrm{ADL}$ operator $A$ is a triple $\left\langle\overline{\mathrm{x}}, \pi_{A}, \epsilon_{A}\right\rangle$, where

- the variables $\overline{\mathrm{x}}$ denote the operator's typed parameters (possibly zero);

- the state formula $\pi_{A}[\overline{\mathbf{x}}]$ denotes the operator's precondition; and

- the effect formula $\epsilon_{A}[\overline{\mathbf{x}}]$ denotes the operator's effects.

The $\Rightarrow$ construct is not to be confused with implication; its purpose is to relate states and successor states. The definition ensures that to the right of the $\Rightarrow$ construct there is always a conjunction of fluent literals. We proceed by defining a normal form for ADL operators; the informed reader should note that this definition deviates from the one used in $[9,13]$.

Definition 5 (Operator Normal Form). An ADL operator $A$ is in normal form if its effect formula has the following syntactic form:

$$
\bigvee_{k}\left(\forall \overline{\mathbf{x}}_{k}\right) \phi_{k}\left[\overline{\mathbf{x}}_{k}\right] \Rightarrow \delta_{k}\left[\overline{\mathbf{x}}_{k}\right]
$$

where $\phi_{k}\left[\overline{\mathbf{x}}_{k}\right]$ is a state formula with free variables among $\overline{\mathbf{x}}_{k}$ and $\delta_{k}\left[\overline{\mathbf{x}}_{k}\right]$ is a conjunction of fluent literals with free variables among $\overline{\mathbf{x}}_{k}$. Further, we require that all $\phi_{k}$ are mutually exclusive.

The following proposition states that we lose nothing by making this operator normal form mandatory:

Proposition 1 (Operator Normal Form). For every effect formula there exists an equivalent effect formula in normal form.

Proof (Sketch). The key observation is that we can always replace e.g. $\top \Rightarrow \delta_{1} \wedge \phi \Rightarrow \delta_{2}$ by the formula $\left(\phi \Rightarrow \delta_{1} \wedge \delta_{2}\right) \vee\left(\neg \phi \Rightarrow \delta_{1}\right)$.

Observe that rewriting an operator to normal form may introduce an exponential blowup.

Example 2 (Blocks World ADL Operator). The following is an ADL operator in normal form for the action Move(block, $x, y$ ) in the blocks world:

$$
\begin{aligned}
\text { Precondition: } & \text { On }\left(\text { block }_{1}, x\right) \wedge x \neq y \wedge \\
& \left(\neg \exists \text { block }_{2}\right) \text { On }\left(\text { block }_{2}, \text { block }_{1}\right) \wedge \\
& \left(\neg \exists \text { block }_{3}\right)\left(\text { On }_{\left.\left(\text {block }_{3}, y\right) \vee y=\text { Table }\right)}\right. \\
\text { Effects: } & \top \Rightarrow{\text { On }\left(\text { block }_{1}, x\right) \wedge \neg \text { On }\left(\text { block }_{1}, y\right)}, x
\end{aligned}
$$


Definition 6 (ADL Problem Descriptions). An ADL planning problem consists of:

- an ADL operator in normal form for each operator name;

- an initial state specification in the form of a conjunction of ground fluent literals; and

- a goal description in the form of a closed state formula.

The following is an ADL problem description analogous to the Fluent Calculus domain from example 1:

Example 3 (ADL Blocks World Description). The only operator shall be as given in example 2 above. Let the initial state be specified by On $\left(\right.$ Block $_{1}$, Table $) \wedge$ On $\left(\right.$ Block $_{1}$, Table $)$ and the goal consist of stacking up all blocks, axiomatized as ( $\exists$ !block)On(block, Table).

ADL admits both open and closed world reasoning - in the open world case the truth-value of fluent literals may be unknown. The existing state transition semantics for ADL from [10], however, is based on the closed world assumption. In this setting the initial state specification is a conjunction of ground fluent atoms. This specification is completed by adding the negation of every ground fluent atom that does not yet occur in the initial state specification, so that eventually every ground fluent atom of the language or its negation occurs in the initial state specification.

The semantics of ADL also makes strong assumptions about the meaning of the constants $\mathcal{C}$ : no two constants denote the same object (uniqueness of names) and all existing objects are named by some constant. This latter requirement allows for substitutional quantification: e.g. a subformula $(\forall x) P(x)$ can equivalently be written as $\bigwedge_{C_{i}} P\left(C_{i}\right)$ where the $C_{i}$ are all the constants of the domain. Thus, although ADL uses the language of first order logic it does not employ first order semantics.

A plan for an ADL planning problem is a ground sequence $\left\langle A_{1}\left(\overline{\mathbf{t}}_{1}\right), \ldots, A_{n}\left(\overline{\mathbf{t}}_{n}\right)\right\rangle$ of operators $A_{i}$ with constants $\overline{\mathbf{t}}_{i}$ substituted for the parameters $\overline{\mathbf{x}}_{i}$. A plan is a solution for the planning problem iff the state obtained by sequentially applying the operators $A_{i}\left(\overline{\mathbf{t}}_{i}\right)$ to the initial state yields a state satisfying the goal description.

\subsection{Plan Constraints}

Plan constraints allow to express both hard and soft constraints on the computed plans: "hard" means that a constraint has to be satisfied while "soft" means that it should, if possible.

State Trajectory Constraints State trajectory constraints are the hard constraints. They allow to express that some property has to hold throughout/at some point/etc. in the plan.

Formally, state trajectory constraints are handled by introducing modalities that can be used in goal descriptions. The available modalities are at end, always, sometime, at-most-once, sometime-after, and sometime-before. We omit the modalities within and always-within since these require an explicit notion of time that is not supported by the ADL subset of PDDL. The modalities may 
not be nested. They can be combined by logical conjunction and be universally quantified from the outside. Universally quantified constraints only serve as shorthand for the equivalent ground formula. ${ }^{5}$ For example we can enforce that a property $\phi$ holds throughout a plan that achieves the goal $\psi$ by writing $\psi \wedge$ always $\phi$.

The original semantics for state trajectory constraints in PDDL has been defined in terms of sequences of state-timepoint pairs $\left\langle\left(S_{0}, 0\right),\left(S_{1}, t_{1}\right), \ldots,\left(S_{n}, t_{n}\right)\right\rangle$, where the $S_{i}$ denote all the states that occur during plan execution in chronological order. In the case of ADL this can be simplified to sequences of states $\left\langle S_{0}, \ldots, S_{n}\right\rangle$.

Definition 7 (Semantics of Temporal Modalities). The semantics of the temporal modalities is then as follows:

$$
\begin{array}{lll}
\left\langle S_{0}, \ldots, S_{n}\right\rangle \vDash \phi & \text { iff } & S_{n} \vDash \phi \\
\left\langle S_{0}, \ldots, S_{n}\right\rangle \vDash \text { at end } \phi & \text { iff } & S_{n} \vDash \phi \\
\left\langle S_{0}, \ldots, S_{n}\right\rangle \vDash \text { always } \phi & \text { iff } & \forall i: 0 \leq i \leq n: S_{i} \vDash \phi \\
\left\langle S_{0}, \ldots, S_{n}\right\rangle \vDash \text { sometime } \phi & \text { iff } & \exists i: 0 \leq i \leq n: S_{i} \vDash \phi \\
\left\langle S_{0}, \ldots, S_{n}\right\rangle \vDash \text { at-most-once } \phi & \text { iff } & \forall i: 0 \leq i \leq n: \text { if } S_{i} \vDash \phi \text { then } \\
& & \neg \exists j, k: i<j<k \leq n: \\
& & S_{j} \vDash \neg \phi \text { and } S_{k} \vDash \phi \\
\left\langle S_{0}, \ldots, S_{n}\right\rangle \vDash \text { sometime-after } \phi \psi & \text { iff } & \exists i: 0 \leq i \leq n: S_{i} \vDash \phi \text { implies } \\
& & \exists j: i<j \leq n: S_{j} \vDash \psi \\
\left\langle S_{0}, \ldots, S_{n}\right\rangle \vDash \text { sometime-before } \phi \psi \text { iff } & \exists i: 0 \leq i \leq n: S_{i} \vDash \phi \text { implies } \\
& & \exists j: 0 \leq j<i: S_{j} \vDash \psi
\end{array}
$$

The expression at-most-once $\phi$ prohibits that $\phi$ changes its truth-value to false and back to true in the course of a plan. A constraint using the always modality might conflict with the initial state specification; we tacitly assume that initial state specifications $\phi$ do not violate any constraints. State trajectory constraints can appear both in the planning problem file and in the action domain file [4].

Preferences Preferences are the soft constraints, i.e. properties that are desired but not required to hold. Instead of an elaborate qualitative model of preferences PDDL adopts a quantitative model.

The syntax for ADL preferences is

$$
\text { preference } \phi \text {, }
$$

where $\phi$ denotes a state formula possibly containing state trajectory constraints. ${ }^{6}$ As in the case of state trajectory constraints preferences may not be nested, and only be combined by logical conjunction. Again, formulas of the form

$$
(\forall \overline{\mathbf{x}}) \text { preference } \phi(\overline{\mathbf{x}})
$$

may be used as shorthand for the logical equivalent conjunction

$$
\bigwedge_{\overline{\mathbf{t}}} \text { preference } \phi(\overline{\mathbf{t}})
$$

\footnotetext{
${ }^{5}$ Recall that ADL admits substitutional quantification.

${ }^{6}$ But note that state trajectory constraints may not contain preferences.
} 
where $\overline{\mathbf{t}}$ denotes all possible ground substitutions for the variables $\overline{\mathbf{x}}$. ADL preferences may occur in goals and in operator preconditions. In the latter case they must not contain the state trajectory modalities.

The semantics of preferences is simple and intuitive. A preference simply always evaluates to true. However, a preference can be satisfied or violated. Let $\left\langle S_{0}, \ldots, S_{n}\right\rangle$ denote the sequence of states corresponding to a plan. If the precondition of the operator applied to state $S_{i}$ contains a preference preference $\phi$, then the preference is violated if $\left\langle S_{i}\right\rangle \vDash \neg \phi$. Likewise a preference occurring in the planning goal is violated if $\left\langle S_{0}, \ldots, S_{n}\right\rangle \vDash \neg \phi$. An overall penalty is assigned to the plan and equals the sum of

- the number of preference $\phi$ expressions from operator preconditions that have been violated; and

- the number of preference $\phi$ expressions from the goal description that have been violated.

The optimal plan in the setting of ADL with plan constraints is the plan with the minimal number of preferences violated. It is worth pointing out that the notion of optimality crucially depends on complete information - in the case of open world ADL it may be impossible to identify whether a plan is optimal.

\section{The Fluent Calculus Semantics for ADL with Plan Constraints}

The Fluent Calculus semantics for ADL with plan constraints is obtained by correctly embedding the latter into the former.

\subsection{Scope of the ADL Constraints}

First off we have to decide whether the constraints from the action domain file and the planning problem file should be treated alike or not. Quoting from [4], "constraints (...) specified in the action domain file (...) might be seen as safety conditions (...) that must alway be respected in any valid plan for the domain (...)." This seems to suggest that these constraints are intended to serve a purpose similar to that of domain constraints in the Fluent Calculus. However, quoting from [15] constraints from the planning problem file "(...) are added to those (if any) in the domain file and together they represent a collection of goals that must be satisfied by any valid plan.". So we adopt the viewpoint that the constraints apply only to the planning goal and not to the action domain as a whole. Let us illustrate the issue at hand by a small example.

Example 4 (Scope of ADL Constraints). Assume that in the ADL blocks world domain from example 3 the constraint always $\mathrm{On}\left(\mathrm{Block}_{1}\right.$, Table) is part of the action domain file. Let $\Sigma$ denote the Fluent Calculus axiomatization of this domain from example 1. If we extend $\Sigma$ by the domain constraint $(\forall s) \operatorname{Holds}\left(\mathrm{On}\left(\mathrm{Block}_{1}\right.\right.$, Table $\left.), s\right)$ the resulting theory is inconsistent. Instead we extend the reasoning problem $\Sigma \vDash(\exists s) \phi(s)$ to the additionally qualified $\Sigma \vDash(\exists s) \phi(s) \wedge \neg\left(\exists s^{\prime}\right) s^{\prime} \sqsubseteq s \wedge \neg \operatorname{Holds}\left(\operatorname{On}\left(\right.\right.$ Block $_{1}$, Table $\left.), s^{\prime}\right)$ - where $\phi$ denotes the goal description. 


\subsection{The Mapping}

We are finally ready to define the mapping:

A Corresponding Language We start by defining a Fluent Calculus signature based on the ADL signature. In order to simplify the presentation we will assume that ADL problems only include the single type ОВЈЕСт. Our results can easily be reformulated in an appropriately sorted version of the Fluent Calculus, so that this is without loss of generality. Given an ADL problem based on a signature with constants $\mathcal{C}$, operator names $\mathcal{A}$, and fluent predicates $\mathcal{F}$ we create a Fluent Calculus signature with corresponding constants $\mathcal{C}^{\prime}$, functions into sort ACTION $\mathcal{A}^{\prime}$, and functions into sort FLUENT $\mathcal{F}^{\prime}$. In order to deal with preferences we introduce the additional sort NUMBER and extend the foundational axioms $\Sigma_{\text {aux }}$ by an axiomatization of the natural numbers.

Based on this signature we include unique-name-axioms for sort ОВ JECT. Likewise we include a domain closure axiom for sort ОВЈЕСт, that is an axiom of the form:

$$
(\forall x) \bigvee_{i=1 . . n} x=c_{i}
$$

where $x$ is a variable of sort ОВЈЕСТ and the $c_{i}$ denote all object constants of the signature. For dealing with preferences we introduce a special fluent, Penalty $/ 1$, that takes a natural number as argument.

The Initial State Mapping ADL initial state specifications $\phi$ to Fluent Calculus initial state axioms $\phi^{\prime}\left[S_{0}\right]$ is done in the obvious way: replace every fluent $F(\overline{\mathbf{x}})$ in $\phi$ by $\operatorname{Holds}\left(F^{\prime}(\overline{\mathbf{x}}), S_{0}\right)$. Below for an ADL state formula $\psi$ by $\psi^{\prime}[s]$ we will denote the corresponding Fluent Calculus state formula obtained in this fashion for an arbitrary situation $s$. Finally we include $\operatorname{Holds}\left(\operatorname{Penalty}(0), S_{0}\right)$ into $\phi\left[S_{0}\right]$. The purpose of this fluent will be to accumulate the number of preferences violated.

The Operators Mapping ADL operators to Fluent Calculus consists of creating corresponding precondition and effect axioms. First we introduce a bit of notation. Let $A$ be an operator with operator precondition $\pi_{A}$. Without loss of generality we assume that $\pi_{A}$ is of the form $\pi_{A_{1}} \wedge \pi_{A_{2}}$ where $\pi_{A_{1}}$ is an ordinary ADL precondition and $\pi_{A_{2}}$ is a conjunction of preferences. Then denote

- by $\Pi_{A_{\text {pref }}}$ the set consisting of the logical parts $\psi_{i}$ of the preferences preference $\psi_{i}$ from $\pi_{A_{2}}$; and

- by $\Pi_{A_{\text {pref.cases }}}$ the set of pairs $\left\langle\bigwedge_{i}(\neg) \psi_{i}, n_{j}\right\rangle$ where each $\psi_{i} \in \Pi_{A_{\text {pref }}}$ and $n_{j} \in \mathbb{N}$ equals the number of $\neg \psi_{i}$ that occur in $\bigwedge_{i}(\neg) \psi_{i}$ where $\psi_{i} \in \Pi_{A_{\text {pref }} \text {. }}$.

That is to say, $n_{j}$ denotes the number of preferences that are violated if $\bigwedge_{i}(\neg) \psi_{i}$ holds. There are $2^{i}$ such pairs in $\Pi_{A_{\text {pref-cases }}}$ and by construction these pairs are mutually exclusive.

For every ADL operator $A=\left\langle\overline{\mathbf{x}}, \pi_{A}, \epsilon_{A}\right\rangle$ from the planning problem we define the action precondition axiom

$$
(\forall \overline{\mathbf{x}}, s) \operatorname{Poss}\left(A(\overline{\mathbf{x}}), s_{1}, s_{2}\right) \equiv \pi_{A_{1}}^{\prime} \wedge s_{2}=\operatorname{Do}(A(\overline{\mathbf{x}}), s) .
$$


Recall that we assume $\epsilon_{A}$ to be of the form $\bigvee_{k}\left(\forall \overline{\mathbf{x}}_{k}\right) \phi\left[\overline{\mathbf{x}}_{k}\right] \Rightarrow \delta\left[\overline{\mathbf{x}}_{k}\right]$. By $\Delta^{+}\left(\Delta^{-}\right)$ denote the set of positive (negative) literals from $\delta\left[\overline{\mathbf{x}}_{k}\right]$. Define the corresponding Fluent Calculus effect axiom as:

$$
\begin{aligned}
& (\forall) \operatorname{Poss}\left(A(\overline{\mathbf{x}}), s_{1}, s_{2}\right) \supset \\
& \bigvee_{k_{i}} \phi^{\prime}\left[\overline{\mathbf{x}}_{k}, s_{1}\right] \wedge \gamma_{i}^{\prime}\left[s_{1}\right] \wedge \operatorname{Holds}\left(\operatorname{Penalty}\left(n_{1}\right), s_{1}\right) \wedge n_{2}=n_{1}+n_{i} \wedge \\
& \llbracket(\forall f)\left[f=\operatorname{Penalty}\left(n_{2}\right) \vee \bigvee_{F(\overline{\mathbf{x}}) \in \Delta^{+}} f=F(\overline{\mathbf{y}})\right] \vee \\
& {\left[\operatorname{Holds}\left(f, s_{1}\right) \wedge f \neq \operatorname{Penalty}\left(n_{1}\right) \wedge \bigwedge_{F(\overline{\mathbf{x}}) \in \Delta^{-}} f \neq F(\overline{\mathbf{x}})\right]} \\
& \equiv \operatorname{Holds}\left(f, s_{2}\right) \rrbracket,
\end{aligned}
$$

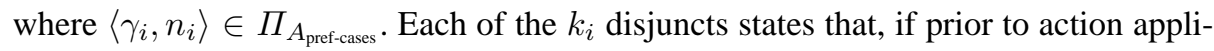
cation

- the accumulated penalty equates $n_{1}$; and

- case $k$ of the ADL operator applies,

then after action application a fluent $f$ holds if-and-only if

- $f$ is equal to Penalty $(n 2)$ where $n 2$ is the new accumulated penalty; or

- $f$ is a positive effect of the ADL operator; or

- $f$ does not equal Penalty $(n 1)$; or

- $f$ held prior to action application and is not a negative effect of the ADL operator.

Let us stress that all the $k_{i}$ disjuncts are mutually exclusive. This completes the definition of a Fluent Calculus domain $\Sigma$ corresponding to an ADL planning problem.

The Goal Descriptions We now turn to goal descriptions. These will be mapped to Fluent Calculus queries that will be evaluated with regard to the domain axiomatization $\Sigma$. If the ADL goal description $\phi$ does not contain any constraints our task is easy: we simply ask whether

$$
\begin{aligned}
\Sigma \vDash & (\exists s, n) \phi^{\prime}[s] \wedge \operatorname{Holds}(\operatorname{Penalty}(n), s) \wedge \\
& \left(\neg \exists s^{\prime}, n^{\prime}\right) \phi^{\prime}\left[s^{\prime}\right] \wedge \operatorname{Holds}\left(\operatorname{Penalty}\left(n^{\prime}\right), s^{\prime}\right) \wedge n^{\prime}<n .
\end{aligned}
$$

We proceed by extending this mapping to goal descriptions containing constraints. Without loss of generality we can assume that the goal description $\phi$ is of the form $\phi_{1} \wedge \phi_{2} \wedge \phi_{3}$, where

- $\phi_{1}$ is an ordinary ADL goal;

- $\phi_{2}$ is a conjunction of state trajectory constraints; and

- $\phi_{3}$ is a conjunction of preferences.

The corresponding Fluent Calculus query is of the form

$$
\left(\exists s, n, n_{\text {final }}\right) \psi_{1}[s, n] \wedge \psi_{2}[s] \wedge \psi_{3}\left[s, n, n_{\text {final }}\right],
$$

where $\psi_{1}[s, n]$ 
- is the formula from (1) if there are no preferences $\phi_{3}$ in the goal description;

- is $(\exists s, n) \phi^{\prime}[s] \wedge \operatorname{Holds}(\operatorname{Penalty}(n), s)$ otherwise.

The mapping from the hard state trajectory constraints $\phi_{2}$ to $\psi_{2}[s]$ can be obtained from the base cases depicted in figure 1 .

\begin{tabular}{|l|l|}
\hline ADL constraint & Fluent Calculus subquery \\
\hline \hline at end $\psi$ & $\psi^{\prime}[s]$ \\
\hline always $\psi$ & $\left(\forall s_{1}\right) s_{1} \sqsubseteq s \supset \psi^{\prime}\left[s_{1}\right]$ \\
\hline sometime $\psi$ & $\left(\exists s_{1}\right) s_{1} \sqsubseteq s \wedge \psi^{\prime}\left[s_{1}\right]$ \\
\hline at-most-once $\psi$ & $\left(\exists s_{1}\right) s_{1} \sqsubseteq s \wedge \psi^{\prime}\left[s_{1}\right] \supset$ \\
& $\neg\left(\exists s_{2}, s_{3}\right) s_{1} \sqsubseteq s_{2} \wedge s_{2} \sqsubseteq s_{3} \wedge s_{3} \sqsubseteq s \wedge \neg \psi^{\prime}\left[s_{2}\right] \wedge \psi^{\prime}\left[s_{3}\right]$ \\
\hline sometime-after $\psi_{1} \psi_{2}$ & $\left(\exists s_{1}\right) s_{1} \sqsubseteq s \wedge \psi_{1}^{\prime}\left[s_{1}\right] \supset\left(\exists s_{2}\right) s_{1} \sqsubseteq s_{2} \wedge s_{2} \sqsubseteq s \wedge \psi_{2}^{\prime}\left[s_{2}\right]$ \\
\hline sometime-before $\psi_{1} \psi_{2}$ & $\left(\exists s_{1}\right) s_{1} \sqsubseteq s \wedge \psi_{1}^{\prime}\left[s_{1}\right] \supset\left(\exists s_{2}\right) s_{2} \sqsubseteq s_{1} \wedge \psi_{2}^{\prime}\left[s_{2}\right]$ \\
\hline
\end{tabular}

Fig. 1. Mapping State Trajectory Constraints to Fluent Calculus.

For the mapping from the preferences $\phi_{3}=\bigwedge_{k}$ preference $\varphi_{k}$ in the goal description to the Fluent Calculus subquery $\psi_{3}\left[s, n, n_{\text {final }}\right]$ we introduce again some notation: denote by $\Phi_{\text {cases }}$ the set of pairs $\left\langle\bigwedge_{k}(\neg) \varphi_{k}, n_{k}\right\rangle$ where $n_{k} \in \mathbb{N}$ equals the number of $\neg \varphi_{k}$ that occur in $\bigwedge_{k}(\neg) \varphi_{k}$. Without loss of generality we assume that $\varphi_{k}$ is of the form $\varphi_{k_{1}} \wedge \varphi_{k_{2}}$, where $\varphi_{k_{1}}$ is an ADL state formula and $\varphi_{k_{2}}$ is a conjunction of state trajectory constraints. We map $\varphi_{k_{1}}$ to $\varphi_{k_{1}}^{\prime}[s]$ and $\varphi_{k_{2}}$ to $\varphi_{k_{2}}^{*}[s]$ - where $\varphi_{k_{2}}^{*}[s]$ is obtained analogously to the mapping from $\phi_{2}$ to $\psi_{2}[s]$. With a little abuse of notation we denote $\varphi_{k_{1}}^{\prime}[s] \wedge \varphi_{k_{2}}^{*}[s]$ by $\varphi_{k}^{\prime}[s]$.

We now define the Fluent Calculus subquery $\psi_{3}\left[s, n, n^{\prime}\right]$ corresponding to $\phi_{3}$ to be

$$
\begin{aligned}
& \left.\bigwedge_{i} \bigwedge_{k}(\neg) \varphi_{k}^{\prime}[s] \supset n_{\text {final }}=n+n_{i}\right) \wedge \\
& \left(\neg \exists s^{\prime}, n^{\prime}, n_{\text {final }}^{\prime}\right) \\
& \bigwedge_{1}\left[s^{\prime}, n^{\prime}\right] \wedge \psi_{2}\left[s^{\prime}\right] \wedge \\
& \left.\bigwedge_{i} \bigwedge_{k}(\neg) \varphi_{k}^{\prime}\left[s^{\prime}\right] \supset n_{\text {final }}^{\prime}=n^{\prime}+n_{i}\right) \wedge n_{\text {final }}^{\prime}<n_{\text {final }},
\end{aligned}
$$

where $\left\langle\bigwedge_{k}(\neg) \varphi_{k}, n_{i}\right\rangle \in \Phi_{\text {cases }}$. This subquery ensures plan optimality by requiring that

- $n_{\text {final }}$ is the sum of

- the penalty $n$ that stems from violated preferences in action preconditions and

- the number $n_{i}$ of preferences $\varphi_{k}$ from the goal description violated by $s$; and

- there does not exists a situation $s^{\prime}$ satisfying the goal description $\psi_{1}$ and the hard plan constraints $\psi_{2}$ with a smaller final penalty.

\subsection{Correctness of the Translation}

We have defined a mapping from ADL planning problems with plan constraints to Fluent Calculus domain axiomatizations $\Sigma$ and Fluent Calculus queries $(\exists s) \phi[s]$. We are now ready to state our main result: 
Theorem 1 (Correctness of the Translation). Let the Fluent Calculus domain $\Sigma$ and query $(\exists s) \phi[s]$ be obtained from an $A D L$ planning problem via our mapping. A sequence $\left\langle A_{1}\left(\overline{\mathbf{t}}_{1}\right), \ldots, A_{n}\left(\overline{\mathbf{t}}_{n}\right)\right\rangle$ of ground $A D L$ operators $A_{i}\left(\overline{\mathbf{t}}_{i}\right)$ is an optimal solution for the planning problem if and only if $\Sigma \vDash \phi\left[D o\left(A_{n}\left(\overline{\mathbf{t}}_{n}\right), D o\left(A_{n-1}\left(\overline{\mathbf{t}}_{n-1}\right), \ldots S_{0}\right) \ldots\right)\right]$.

Proof (Sketch). The full proof of this theorem is quite tedious and therefore omitted. However, in order to provide some evidence for the correctness of the theorem, we point out that our embedding is very generic, in the sense that the Fluent Calculus domain axiomatization $\Sigma$ and the ADL planning problem correspond axiom-by-axiom.

\section{Summary}

\subsection{Related Work}

The series of works [7-9] successively provided logical semantics for more and more expressive fragments of PDDL by interpreting these in a recently proposed first order modal variant of the Situation Calculus [16]. None of these works covers plan constraints yet. Many ontological features of PDDL like e.g. concurrent actions and actions with duration are not present in the basic Situation Calculus, however. Thus, in order to obtain a mapping from PDDL fragments to Situation Calculus the underlying logic had to be considerably extended. For Fluent Calculus such extensions have first been introduced in [17]. Most likely these ideas can be adapted in order to obtain Fluent Calculus semantics for equally expressive fragments of PDDL as those covered in [7-9].

Using a mapping defined in [13] we can obtain Situation Calculus axiomatizations corresponding to their Fluent Calculus counterparts. This immediately yields a Situation Calculus semantics for ADL with plan constraints.

Instead of plan constraints costs associated to actions have been introduced for the sequential deterministic part of this year's planning competition at ICAPS-08. ${ }^{7}$ This system can also very naturally be interpreted in the Fluent Calculus; plan costs can be computed by summing over situation terms.

Our result identifies a fragment of the Fluent Calculus for which reasoning can be based on efficient specialized planning software instead of the more general constraint logic programming implementation Flux [11]. A tight integration may be achieved by adopting ideas from [18], where planning problems have efficiently been encoded and solved in CLP(FD). Note that reversing our mapping does not introduce an additional blowup as opposed to compiling operators to normal form.

\subsection{Conclusion}

We have given a purely declarative semantics for ADL with plan constraints by interpreting it in the basic Fluent Calculus. Our semantics is logical, as opposed to the only previously available semantics, which was based on state transitions. Along the way we have clarified the role played by state trajectory constraints by determining their scope. The resulting system is expressive and - since both PDDL and the Fluent Calculus are action-centered formalisms - very natural.

\footnotetext{
${ }^{7}$ See http://ipc.informatik.uni-freiburg.de/.
} 


\section{References}

1. Fikes, R.E., Nilsson, N.J.: STRIPS: A New Approach to the Application of Theorem Proving to Problem Solving. Artificial Intelligence 2 (1971) 189-208

2. Pednault, E.P.D.: ADL: Exploring the Middle Ground between STRIPS and the Situation Calculus. In: Proceedings of the First International Conference on Principles of Knowledge Representation and Reasoning (KR 89), San Mateo, California, Morgan Kaufmann (1989) 324-332

3. Ghallab, M., Howe, A., Knoblock, C., McDermott, D., Ram, A., Veloso, M., Weld, D., Wilkins, D.: PDDL-The Planning Domain Definition Language (1998) ftp://ftp.cs.yale.edu/pub/mcdermott/software/pddl.tar.gz.

4. Gerevini, A., Long, D.: Preferences and Soft Constraints in PDDL 3.0. In: Proceedings of the ICAPS-2006 Workshop on Preferences and Soft Constraints in Planning, Lake District of the UK (2006) 46-53

5. Lifschitz, V.: On the Semantics of STRIPS. In: Reasoning about Actions and Plans, Temberline, Oregon, Morgan Kaufmann (1986) 1-9

6. Pednault, E.P.D.: ADL and the State-Transition Model of Action. Journal of Logic and Computation 4 (1994) 467-512

7. Claßen, J., Lakemeyer, G.: A Semantics for ADL as Progression in the Situation Calculus. In: Proceedings of the 11th International Workshop on Non-Monotonic Reasoning (NMR06), Lake District, UK (2006)

8. Claßen, J., Hu, Y., Lakemeyer, G.: A Situation-Calculus Semantics for an Expressive Fragment of PDDL. In: Proceedings of the Twenty-second National Conference on Artificial Intelligence (AAAI 2007), Menlo Park, CA (2007) 956-961

9. Claßen, J., Eyerich, P., Lakemeyer, G., Nebel, B.: Towards an Integration of Golog and Planning. In: Proceedings of the Twentieth International Joint Conference on Artificial Intelligence (IJCAI 07), Hyderabad, India (2007) 1846-1851

10. Fox, M., Long, D.: PDDL2.1: An Extension to PDDL for Expressing Temporal Planning Domains. Journal of Artificial Intelligence Research 20 (2003) 61-124

11. Thielscher, M.: Reasoning Robots: The Art and Science of Programming Robotic Agents. Springer, Dordrecht, NL (2005)

12. Reiter, R.: Knowledge in Action: Logical Foundations for Describing and Implementing Dynamical Systems. MIT Press, Cambridge, MA (2001)

13. Thielscher, M.: A Unifying Action Calculus. Artificial Intelligence (submitted) (2007) www. fluxagent.org/publications.htm.

14. Gelfond, M., Lifschitz, V.: Action Languages. Electronic Transactions on Artificial Intelligence 3 (1998) http: / / www. ep. liu. se/ea/cis/1998/016/.

15. Gerevini, A., Long, D.: BNF Description of PDDL3.0. http://zeus.ing.unibs.it/ipc-5/ (2005)

16. Lakemeyer, G., Levesque, H.J.: Situations, Si! Situation Terms, No! In: Proceedings of the Ninth International Conference on Principles of Knowledge Representation and Reasoning (KR 04), Whistler, Canada (2004) 516-526

17. Thielscher, M.: The Concurrent, Continuous Fluent Calculus. Studia Logica 67 (2001) 315-331

18. Dovier, A., Formisano, A., Pontelli, E.: Multivalued Action Languages with Constraints in CLP(FD). In: Proceedings of the Twenty-third International Conference on Logic Programming (ICLP 2007), Porto, Portugal (2007) 255-270 